\title{
EPILEPSIA PARCIAL ASSOCIADA A TUMORES CEREBRAIS PRIMÁRIOS
}

\author{
Paulo Thadeu Brainer-Lima' ${ }^{1}$, Alessandra Mendes Brainer-Lima², \\ Hildo Azevedo Filho ${ }^{3}$, Arthur Cukiert ${ }^{4}$
}

\begin{abstract}
RESUMO - Objetivo: Estudar as características clínicas da epilepsia em pacientes com tumores cerebrais primários de crescimento lento. Método: Quarenta e dois pacientes com epilepsia refratária ao tratamento medicamentoso associada a tumor cerebral de crescimento lento foram avaliados entre junho de 1992 e setembro de 1999. Resultados: Setenta e cinco por cento dos pacientes tinha menos de 15 anos quando iniciaram-se as crises convulsivas. 0 exame neurológico foi normal em $67,7 \%$ dos pacientes. Predominaram as crises epilépticas do tipo parcial complexa $(90,4 \%)$, com até 16 crises mensais. As crises foram do tipo parcial simples (PS) evoluindo para parcial complexa (PC) em 10 pacientes, PC isoladas em 11 e PC evoluindo para generalizada em 11. Conclusão: Noventa e cinco por cento dos pacientes com tumores cerebrais de crescimento lento apresentaram crises parciais, mesmo que com baixa frequência. Estas últimas podem ser uma manifestação precoce de tumor de crescimento lento nas crianças, mesmo sem nenhuma alteração no exame neurológico.
\end{abstract}

PALAVRAS-CHAVE: epilepsia parcial, tumor cerebral primário, epilepsia refratária.

\begin{abstract}
Partial epilepsy associated to primary brain tumors
ABSTRACT- Objective: To characterize the epileptic syndrome of patients with primary cerebral tumor of slow growth. Method: Clinical evaluation of fourty two patients with refractory epilepsy associated with cerebral tumor of slow growth were operated between June 1992 and September 1999. Results: Almost 75\% of the patients were less than 15 years old when they began the epileptic crises, $67.7 \%$ had normal neurological exam in the first evaluation. More than $90 \%$ of the total had seizures of the partial complex type, frequently inferior to 16 per month. The seizures were partial simple (PS) developing for partial complex (PC) in 10 patient and only PC in 11 Partial complex associated with generalized tonic-clonic seizure (GTC) happened in 11 patients, PS for PC and GTC in 4 patients. Conclusion: The crises of the partial type, with not very high frequency, can suggest a precocious manifestation of the tumor in the children, even with normal neurological exam.
\end{abstract}

KEY WORDS: partial epilepsy, primary cerebral tumor, refractory epilepsy.

A história clínica do paciente epiléptico pode sugerir a presença de tumor cerebral quando há sinais neurológicos focais ou de hipertensão intracraniana, status epilepticus repetidos ou frequentes, paralisias pós- críticas, modificação do padrão das crises já existentes ou refratariedade ao tratamento medicamentoso ${ }^{1}$. Excluindo-se os pacientes com epilepsia parcial benigna geneticamente determinada, aqueles com crises parciais seriam, a priori, portadores de alteração estrutural ou funcional do cérebro, seja ela macro ou microscopicamente verificável².

Considerando todos os grupos etários, os tumo- res cerebrais são causa de aproximadamente $2 \%$ das epilepsias, sendo este percentual de $0,5 \%$ nas crianças e de $10 \%$ nos adultos ${ }^{3}$. Crises epilépticas ocorrem em 35 a $70 \%$ de todos os pacientes com tumor cerebral $^{4}$. O diagnóstico precoce do tumor cerebral de crescimento lento em crianças com epilepsia refratária, mesmo sem alterações no exame neurológico ${ }^{5}$, possibilita tratamento eficaz da epilepsia e do tumor cerebral, evitando suas consequências crônicas.

Neste estudo analisamos as características da epilepsia em pacientes com tumores cerebrais de crescimento lento.

\footnotetext{
${ }^{1}$ Neurocirurgião, Preceptor do Programa de Residência Medica em Neurocirurgia do Hospital da Restauração-SUS-(HR), Mestre em Neuropsiquiatria - Universidade Federal de Pernambuco (UFPE), Doutorando em Cirurgia da UFPE, Recife PE, Brasil; ${ }^{2}$ Radiologista do Centro Diagnóstico Multimagem do Recife, Recife PE, Brasil; ${ }^{3}$ Neurocirurgão, Professor-Doutor do Hospital das Clinicas da UFPE, Coordenador do Serviço de Neurocirurgia do HR, Recife PE, Brasil; ${ }^{4}$ Neurocirurgião, Chefe do Serviço de Neurocirurgia e Cirurgia de Epilepsia do Hospital Brigadeiro de São Paulo, Diretor da Clínica de Epilepsia de São Paulo, São Paulo SP, Brasil.
}

Recebido 8 Fevereiro 2002, recebido na forma final 12 Abril 2002. Aceito 26 Abril 2002. 


\section{MÉTODO}

A presença de epilepsia resistente ao tratamento medicamentoso, associada a tumor cerebral primário de crescimento lento foi o critério de inclusão para 42 pacientes operados. Os pacientes foram avaliados através de exames realizados rotineiramente na investigação de pacientes com epilepsia refratária ao tratamento clínico. Além da epilepsia refratária ao tratamento clínico, todos os pacientes foram selecionados de acordo com a presença de lesões demonstradas na ressonância magnética ou no estudo anátomo-patológico. Depois de operados, somente aqueles com seguimento mínimo de 24 meses e reavaliados em outubro de 2001 foram considerados nesse estudo, em acordo com projeto apresentado a Comissão de Ética do hospital.Foram analisados a idade de início da epilepsia, tipos e frequência das crises, descrição das crises, exame neurológico e resultados pós-operatórios ${ }^{6}$. 0 acompanhamento clínico pós-operatório variou de 24 a 76 meses (média 35,2 meses).

A análise estatística utilizou o teste de qui-quadrado de associação ou o teste exato de Fisher, de acordo com o necessário e significância para $p<0,05$.

\section{RESULTADOS}

Foram estudados 30 homens e 12 mulheres. A idade variou de 6 a 62 anos (média 23,4 anos). Vinte e oito por cento dos pacientes eram menores de 14 anos. O intervalo de tempo, desde o momento das primeiras crises até o achado do tumor cerebral, variou de 2 a 34 anos (média 12,2 anos). Doze pacientes $(28,5 \%)$ levaram 2 a 5 anos para seu diagnóstico, $9(21,4 \%)$ de 6 a 10 anos, $14(33,3 \%)$ de 11 a 20 anos e $8(19,0 \%)$ de 21 a 30 anos. A idade dos pacientes quando iniciadas as crises epilépticas variou de 2 a 52 anos (média 13,0 anos), ocorrendo na primeira década de vida em 24 pacientes $(57,1 \%)$. Em $74,8 \%$ dos pacientes as crises iniciaram-se antes dos 15 anos.

Tabela 1. Tipo das crises epilépticas (CE).

\begin{tabular}{lccc}
\hline Tipo & Frequência & $\%$ & \% Acumulado \\
\hline MS & 1 & 2,9 & 2,9 \\
GEN & 3 & 8,8 & 11,7 \\
PC & 12 & 26,5 & 38,2 \\
PC/GEN & 11 & 32,4 & 70,6 \\
PS/PC & 10 & 20,6 & 91,2 \\
PS/PC/GEN & 5 & 8,8 & 100 \\
Total & 42 & 100 & - \\
\hline
\end{tabular}

PS, parcial simples; PC, parcial complexa; GEN, generalizada; MS, área motor suplementar.
As crises foram do tipo parcial simples (PS) evoluindo para parcial complexa (PC) em 10 pacientes, PC isoladas em 12; PC evoluindo para generalizada (Gen) em 11 e PS evoluindo para PC e Gen em 5 pacientes. Crise de área motora suplementar (MS) foi encontrada em um paciente e somente Gen em três pacientes (Tabela 1). Em 32 pacientes (76,1\%), a PC foi precedida ou não de PS e seguida ou não por Gen. A PS não foi relatada de forma isolada em nenhum paciente. Automatismos orais, precoces ou tardios, aconteceram em 24 pacientes, em 21 deles com tumor cerebral em região temporal (87,5\%). Aura epigástrica foi referida em 9 pacientes, todos com lesão temporal. Em 17 pacientes (40,4\%), houve referência à Gen, em 12 com frequência menor que uma crise por semestre. A maior frequência de Gen foi duas crises diárias, o que ocorreu em um paciente. As Gen ocorreram principalmente nos anos iniciais da epilepsia, após suspensão abrupta da medicação antiepiléptica ou por ingestão de álcool. Ocorreu estado de mal epiléptico em dois pacientes. As crises tornaram-se mais prolongadas ou mais frequentes durante a evolução da epilepsia em $33 \%$ dos pacientes, apesar do uso de medicação antiepiléptica.

A frequência das crises variou de uma a 300 por mês, com média de 52,4 por mês, 17 pacientes $(40,5 \%)$ tendo até 10 crises mensais. Em 27 pacientes $(64,2 \%)$, havia referência a alteração de memória. Todos os pacientes foram submetidos a avaliação neuropsicológica. Vinte e quatro pacientes apresentaram comprometimento da memória visual ou verbal. Em 14 pacientes com lesão à direita, ocorreu déficit de memória visual e, em 10 com lesão à esquerda, houve déficit da memória verbal. Em 11 pacientes $(26,1 \%)$ havia história familiar de epilepsia ou crises isoladas em parentes do primeiro grau. Con-

Tabela 2. Distribuição de frequência da etiologia dos tumores cerebrais primários com epilepsia refratária.

\begin{tabular}{lcc}
\hline Histologia & Frequência & $\%$ \\
\hline Ganglioglioma & 19 & 45,2 \\
Astrocitoma & 13 & 30,1 \\
Oligodendroglioma & 5 & 11,9 \\
Astrocitoma anaplásico & 1 & 2,4 \\
Oligodendroglioma anaplásico & 1 & 2,4 \\
Xantoastrocitoma & 1 & 2,4 \\
Tumor desembrioblástico neuroepitelial & 2 & 4,8 \\
Total & 42 & 100 \\
\hline
\end{tabular}


vulsões febris constavam nos antecedentes de quatro dos 42 pacientes; sofrimento perinatal foi referido em um paciente.

A remoção cirúrgica foi total em $85,3 \%$ dos pacientes. A avaliação pós-operatória, segundo a escala de Engel ${ }^{7}$, mostrou que a maioria $(81,2 \%)$ foi classificada no grau I (sem crises) e os restantes $(18,8 \%)$ no grau II (melhora >90\%).

Os achados anátomo-patológicos dos 42 pacientes operados estão relacionados na Tabela 2 . Todos os diagnósticos foram confirmados histologicamente.

\section{DISCUSSÃO}

A epilepsia refratária é associada a níveis elevados de deterioração psicossocial e morbidade nas crianças e adolescentes, exigindo uma avaliação cirúrgica precoce, principalmente nos tumores cerebrais de crescimento lento. A metade dos pacientes selecionados nesta série tinham até 20 anos, confirmando a predileção desses tumores por pacientes jovens ${ }^{8}$.

O tumor cerebral de crescimento lento mais relacionado com epilepsia refratária em pacientes jovens é o ganglioglioma (GG), pouco encontrado entre os tumores cerebrais em pacientes sem epilepsia refratária9 ${ }^{9}$ Pertencem à categoria dos tumores cerebrais primários com presença de células da glia e neurônios adultos, tendo características patológicas e clínicas quase idênticas aos gangliocitomas, em que, juntos ou em separado, a glia e o neurônio podem apresentar comportamento anaplásico ${ }^{10,11}$.

Morris et al. ${ }^{12}$ relataram que $12 \%$ dos casos operados na Cleveland Clinic, durante 10 anos, foram tumores cerebrais de crescimento lento, todos com menos de 30 crises por mês e mais de 6 anos de história. Kirkpatrik et al. ${ }^{13}$ relataram a média de duração das crises de 10,9 anos para os gliomas de baixo grau (tumor cerebral de crescimento lento), antes de seu diagnóstico efetivo, com média de 30,6 crises por mês. Spencer et al. ${ }^{14}$ descreveram que, nas lesões com alto grau de indiferenciação celular (tumor cerebral de crescimento rápido), existe um tempo de 1 a 3 anos entre o início das crises e a cirurgia, porém com uma frequência bem maior de crises (200/ mês). Realmente, a frequência das crises foi maior entre os portadores de tumor cerebral de crescimento rápido (média 150 crises/mês), diferente da observada no tumor cerebral de crescimento lento (média 30 crises/mês), em todos os trabalhos encontrados, sugerindo uma espera prolongada pela investigação específica quando existem poucas crises. Rasmussen ${ }^{15}$ e Pace et al. ${ }^{16}$ argumentaram que o tumor cerebral de crescimento lento tem grande potencial para provocar crises, devido a maturação progressiva do foco epiléptico. A diminuição do intervalo entre o início das crises e o diagnóstico do tumor cerebral e cirurgias convencionais precoces para o tumor cerebral talvez possam impedir a formação da zona epileptogênica e levar a melhor controle das crises.

Britton et al. ${ }^{17}$ relataram 51 pacientes com epilepsia refratária e tumor cerebral em que $92 \%$ destes sofriam de crise epiléptica parcial. Outros estudos com populações heterogêneas de tumor cerebral de crescimento lento também demonstraram a predominância da crise epiléptica parcial ${ }^{18,19}$. Award et al. ${ }^{20}$ descreveram 47 pacientes com tumor cerebral primário e epilepsia refratária, nos quais a PC não foi específica para a localização anatômica da lesão, podendo ocorrer em lesões temporais ou extra-temporais.

A aura epigástrica foi referida em 9 dos portadores de tumor cerebral temporal $(37,5 \%)$, nos quais predominavam também os automatismos orais ${ }^{21}$. As auras frequentemente persistem depois de ressecções da porção mesial do lobo temporal ${ }^{22}$. Esse fato sugere que elas tenham comportamento semelhante às memórias com distribuição mais difusa e neocortical.

As crises do lobo da ínsula não têm clínica ou eletrofisiologia de fácil interpretação, simulando uma falsa zona epileptogênica temporal, mesmo em lesões completamente insulares ${ }^{24,24}$. A avaliação do exame neurológico mostrou normalidade em $67,7 \%$ dos pacientes. As alterações foram restritas aos efeitos da localização do tumor cerebral.

Spencer et al. ${ }^{14}$, em estudo com 190 pacientes tratados cirurgicamente para crise epiléptica parcial refratária, encontraram 19 pacientes com tumores cerebrais e, 16 desses com tumor cerebral primário. Esses autores ainda chamavam atenção para o grande número de crianças neste grupo, concluindo que em casos de epilepsia parcial refratária deve haver sempre suspeita de tumor cerebral primário de crescimento lento. Entretanto, essa epileptogenicidade denota a incidência de crises em cada tipo de tumor e não seu grau de severidade. Os dois comportamentos distintos, um com história curta com muitas crises e o outro com história longa com poucas crises correspondem a modelos de malignidade ou benignidade do tumor cerebral primário, respectivamente. No tumor cerebral de crescimento lento com epilepsia refratária, a frequência de crises pode ser progressivamente menor com a idade, sem distinção histológica ${ }^{25}$. 
Nas crianças, o tumor cerebral de crescimento lento esteve presente entre $1 \%$ a $9 \%$ dos pacientes com tumores primários, sendo crises epilépticas o principal sintoma em todos os $\operatorname{casos}^{26}$. Entretanto, nenhum desses relatos informaram especificamente quanto à presença de crises epilépticas parciais ou refratárias.

Metade dos pacientes com epilepsia parcial refratária e tumor cerebral primário de crescimento lento tinha até 7 anos e dois-terços até 15 anos de idade quando iniciaram as crises epilépticas; $67 \%$ tinham exame neurológico normal na primeira avaliação e em mais de $90 \%$ do total a crise foi do tipo parcial, com frequencia inferior a 16 crises mensais, sugerindo um início precoce da manifestação do tumor por uma síndrome epiléptica comum nos infantes, sem outros comemorativos considerados específicos na semiologia do tumor cerebral. Os tumores cerebrais de crescimento lento são etiologia relativamente frequente de crises parciais refratárias, em especial em crianças.

\section{REFERÊNCIAS}

1. Weber P, Silbergeld DL, Winn HR. Surgical resection of epileptogenic cortex associated with structural lesions. Neurosurg Clin N Am 1993;2:327-337.

2. Haddad SF, Moore SA, Menezes AH, Vangilder JC. Ganglioglioma: 13 years of experience. Neurosurgery 1992;31:171-178.

3. Tatter S B, Wilson CB, Hars GR. Neuroepithelial tumors of the adult brain. In Youmans JR (ed.). Neurological surgery. 4. Ed, Philadelphia: Saunders,1996:2612-2684

4. Vilemure JG, Tribolet N. Epilepsy in patients with central nervous system tumors. Curr Opin Neurol 1996;9:424-428.

5. Lee TK, Nakasu Y, Jeffre MA, Molyneaux AJ, Adams CB. Indolent glioma: a cause of epilepsy. Arch Dis Child 1989;64:1666-1671.

6. Montgomery DC. Design and analysis of experiments. New York: Jonh Wiley \& Sons, 1984:30-49.

7. Engel J, Van Ness PC, Rasmussen TB, Ojemann LM. Outcome with respect to epileptic seizures. In Engel J Jr(ed). Surgical treatment of epilepsies. New York: Raven Press, 1993:609-621.
8. Johannsson GH, Rakete HL, Roessmann U. Gangliogliomas: pathological and clinical correlation. J Neurosurg 1981;54:58-63.

9. Kalyan-Raman UP, Olivero WC. Ganglioglioma: a corelative clinicopathological and radiological study of 10 surgically treated cases with follow up. Neurosurgery1987;20:428-433.

10. Arriba-Villamor CM, Martinez-Mata A, Espinosa-Mogro H, RubioVigueira V. Ganglion cell tumors. Rev Neurol 1998;27:1008-1011.

11. Dash RC, Provenzale JM, Mccomb RD, Perry DA, Longee DC, Mclendon RE. Malignant supratentorial ganglioglioma (ganglioncell-giant cell glioblastoma): a case report and review of the literature. Arch Pathol Lab Med 1999;123:342-345.

12. Morris HH, Estes ML, Gilmore R, Van Ness PC, Barnett GH, Turnbull J. Chronic intractable epilepsy as the only symptom of primary brain tumor. Epilepsia 1993;34:1038- 1043.

13. Kikpatrick PJ, Honavar M, Janota I, Polkey CE. Control of temporal lobe epilepsy following en bloc resection of low grade tumors. J Neurosurg 1993;78:19-25.

14. Spencer DD, Spencer SS, Mattson RH. Intracerebral masses in patients with intractable partial epilepsy. Neurology 1984;34:432-436.

15. Rasmussen T. Surgery of epilepsy associated with brain tumors. In Purpura DP, Penry JK, Walter RD. (eds.) Neurosurgical management of the epilepsies. New York: Raven Press, 1975:227-229.

16. Pace A, Bove L, Innocenti $P$, et al. Epilepsy and gliomas: incidence and treatment in 119 patients. J Exp Clin Cancer Res 1998;17:479-482.

17. Britton JW, Cascino GD, Sharbroligh FW, Kelly PJ. Low grade glial neoplasms and intractable partial epilepsy: efficacy of surgical treatment. Epilepsia 1994;35:1130-1135.

18. Brainer-Lima PT, Rao S, Cukiert A, Gronisch G, Yacubian EM, Marino $\mathrm{R}$ Jr. Surgical treatment of refractory epilepsy associated with space occupying lesions: experience and review. Arq Neuropsiquiatr 1996;54:384-392.

19. Fried I, Spencer DD, Spencer SS. The anatomy of epileptic auras: focal pathology and surgical outcome. J Neurosurg 1995;83:60-66.

20. Award IA, Rosenfeld J, Ahal J, Hahn JF, Luders H. Intractable epilepsy and structural lesions of the brain: mapping, resection strategies, and seizure outcome. Epilepsia 1991;32 :179-186.

21. Gil-Nagel A, Risinger MW. Ictal semiology in hippocampal versus extrahippocampal temporal lobe epilepsy. Brain 1997;120:183-192.

22. Fried I. Auras and experimental responses arising in the temporal lobe. J Neuropsychiat Clin Neurosci 1997;9:420-428.

23. Cukiert A, Forster C, Andrioli MS, Rayman L. Insular epilepsy: similarities to temporal lobe epilepsy. Case report. Arq Neuropsiquiatr 1998;56:126-128.

24. Savic I, Bookheimer, SY, Fried I, Engel J Jr. Olfactory bedside test: a simple approach to identify temporo-orbitofrontal dysfunction. Arch Neurol 1997;54:62-168

25. Lote K, Stenwing AE, Skullerud K. Prevalence and prognostic significance of epilepsy in patients with gliomas. Eur Cancer 1998;34:98-102.

26. Johnson JH, Hariharan S, Berman J, et al. Clinical outcome of pediatric gangliogliomas: ninety-nine cases over 20 years. Pediatr Neurosurg 1997;27:203-207. 